\title{
Aspectos paleobiológicos de dos ejemplares de Mammuthus columbi (Mammalia, Proboscidea, Elephantidae) del Pleistoceno de Puebla, centro de México
}

Gerardo Garbot-Ghanona, Zaid Lagunas-Rodríguez, Francisco Javier Jiménez-Moreno, Sergio Suárez

Gerardo Carbot-Chanona

gfcarbot@gmail.com

Museo de Paleontología "Eliseo Palacios Aguilera". Calzada de Los Hombres Ilustres s/n, Antiguo Parque Madero, C.P. 29000, Tuxtla Gutiérrez, Chiapas.

\section{Zaid Lagunas-Rodríguez}

Sergio Suáerz

Centro INAH-Puebla, Avenida Ejércitos de Oriente s/n, Centro Cívico 5 de mayo, Los Fuertes, C.P. 72270, Puebla, Puebla.

Francisco Javier Jiménez-Moreno Posgrado en Ciencias Ambientales, Edif 103 D (planta baja), Benemérita Universidad Autónoma de Puebla, Col. Jardines de San Manuel, C.P. 72570, Puebla, México.

BOL. SOC. GEOL. MEX. 2017

VOL. 69 NO. 3

P. $591-609$

\section{RESUMEN}

Se describen formalmente dos ejemplares de Mammuthus columbi (CRINAH-2274 y CRINAH-2275), con base en elementos craneales, apendiculares y molares aislados, procedentes de dos localidades del Pleistoceno de la parte centro-este de Puebla y se comentan algunos aspectos paleobiológicos. La asignación específica de los ejemplares se basa principalmente en las características de los M3, los cuales exhiben una frecuencia lamelar de 5 a 7 , esmalte fuertemente crenulado con un grosor de 1.5 a 3.8 mm y poseen de 20 a 22 láminas. Se estimó la edad de ambos individuos; el ejemplar CRINAH-2274 tiene 34 AEA, mientras que la edad estimada para CRINAH-2275 fue de 37 a 41 AEA. Sólo pudo establecerse el sexo de CRINAH-2274, el cual es un macho. Por último, se realizó un análisis de ángulos de mesodesgaste para caracterizar la dieta y el hábitat; este método se usa por primera vez en México. Nuestros resultados muestran que ambos individuos eran estrictamente pacedores $\left(>130^{\circ}\right)$ con una dieta basada principalmente en pastos. Lo anterior sugiere la existencia de hábitats abiertos en la región centro-este de Puebla durante el Pleistoceno.

Palabras clave: Mamuts, paleobiología, ángulos de mesodesgaste, preferencia de hábitat, Puebla.

\section{ABSTRACT}

Two specimens of Mammuthus columbi from two Pleistocene localities in the central-eastern of Puebla are formally described (CRINAH-2274 and CRINAH-2275), based on cranial, appendicular elements and isolated molar, and some paleobiological aspects are discussed. The specific assignment of the specimens is based mainly on the characteristics of the M3, which exhibit a lamellar frequency of 5 to 7, strongly crenulated enamel with a thickness of 1.5 to $3.8 \mathrm{~mm}$ and has 20 to 22 plates. The age of both individuals was estimated; the CRINAH-2274 specimen has 34 AEI, while the estimated age for CRINAH-2275 was 37 to 41 AEr. Only the sex of CRINAH-2274 could be established, which is a male. Lastly, mesowear angle analysis was carried out to characterize the diet and habitat; this method is used for the firts time in Mexico. Our results show that both specimens were strictly grazers $\left(>130^{\circ}\right)$ with a diet based mainly on grasses. This suggests the existence of open habitats in the central-eastern region of Puebla in the Pleistocene.

Keyzords: Mamooths, paleobiology, mesozvear angle, habitat preference, Puebla. 


\section{Introducción}

El Orden Proboscidea actualmente incluye tres especies, el elefante africano de sabana Loxodonta africana, el elefante africano de bosque Loxodonta cyclotis y el elefante asiático Elephas maximus, todas incluidas en la familia Elephantidae (Shoshani, 2005). Este orden fue mucho más diverso en el pasado, ya que se han contabilizado 175 especies y subespecies, clasificadas en 42 géneros y 10 familias (Shoshani y Tassy, 2005). Proboscidea tuvo su origen en el Paleoceno tardío, a lo largo de la costa del Mar Tethys en el norte de África y sur de Asia, se extendió a Eurasia durante el Mioceno temprano y, posteriormente, alcanzó América del Norte vía Beringia en el Mioceno tardío (Lambert y Shoshani, 1998).

Proboscidea está representado en el Pleistoceno de México por tres familias, Mammuthidae, con un sólo género: Mammut; Gomphotheriidae, con dos géneros: Cuvieronius y Stegomastodon y Elephantidae, con un género: Mammuthus (Arroyo-Cabrales et al., 2010). De estos, Mammuthus es el más comúnmente encontrado a lo largo y ancho del territorio mexicano. En la literatura se mencionan cinco especies para México: Mammuthus columbi, M. hayi, M. imperator, M. primigenius y $M$. sonoriensis (Arroyo-Cabrales et al., 2003b). Actualmente sólo se reconocen dos especies, el llamado complejo Mammuthus hayi/ meridionalis que se conoce de localidades del Pleistoceno temprano (Irvingtoniano) en el norte y centro del país y Mammuthus columbi, que se encuentra en sedimentos del Pleistoceno tardío (Rancholabreano) a lo largo y ancho de México, desde localidades norteñas como El Carrizal, en Baja California Sur (Rochín-Bañaga et al., 2017), hasta la más sureña ubicada en El Amate, en Chiapas (Gómez-Pérez y Carbot-Chanona, 2012), siendo Yucatán y Tabasco los únicos estados sin reportes hasta el momento (Arroyo-Cabrales et al., 2010). Mammuthus columbi (el mamut de Cristoforo Colombo, en referencia al Nuevo Mundo) fue descrito por Hugh Falconer (1857) bajo el nombre de Elephas columbi. Esta especie se distribuyó desde Canadá hasta Centroamérica (Kurtén y Ander- son, 1980; Alvarado, 1986; Laurito, 1988; Lucas et al., 1997; Laurito y Aguilar, 2006) y se cree evolucionó en América del Norte a partir de un primitivo inmigrante euroasiático, similar a Mammuthus trogontherii (Lister y Sher, 2015).

\subsection{REGISTROS DE MAMMUTHUS EN PUEBLA}

Los primeros restos de Mammuthus inequívocamente identificados, provenían de las localidades de San Francisco Totimehuacan y San Baltazar Tétela, y estuvieron resguardados en el gabinete del antiguo Colegio del estado, hoy Benemérita Universidad Autónoma de Puebla (Armenta-Camacho, 1978). Posteriormente, se adicionaron registros de Mammuthus gracias a los trabajos de Félix y Lenk (1891), Osborn (1905, 1942), Freudenberg (1922), Armenta-Camacho (1957, 1978), Irwin-Williams (1967), Silva-Bárcenas (1969), Lagunas-Rodríguez y Suárez-Cruz (1997), Suárez-Cruz y Lagunas-Rodríguez (2008), Lagunas-Rodríguez et al. (2013), entre otros.

En la última década, en Puebla se han reportado hallazgos de mamuts cuyos restos son expuestos como consecuencia de la construcción de caminos y edificaciones, siendo la mayoría atendidos por personal del Instituto Nacional de Antropología e Historia, delegación Puebla (Lagunas-Rodríguez y Suárez-Cruz, 1997; Lagunas-Rodríguez et al., 2013). En junio de 2007 y marzo de 2013, se atendieron las denuncias sobre el hallazgo de restos fósiles en barranca El Morillo, al norte de Ciudad Serdán, municipio de Chalchicomula de Sesma y en la colonia Las Cruces, municipio de Amozoc. En ambos casos, se rescataron restos óseos de Mammuthus que son el motivo del presente manuscrito.

A pesar de que los registros de mamuts son frecuentes en México y los restos abundantes, poco de ese material ha sido descrito formalmente, por lo que se desconocen las variaciones intraespecíficas entre poblaciones, preferencia de hábitat o aspectos generales sobre su paleobiología, como la edad y el sexo. Por tal motivo, el objetivo de este trabajo es describir formalmente el material de Mammuthus encontrado en el centro-este de Pue- 
bla, así como aportar datos sobre algunos aspectos biológicos de los individuos, como edad, sexo, dieta y preferencia de hábitat, información que sin duda contribuirá a entender mejor la paleobiología de los mamuts en México.

Abreviaturas institucionales: CRINAH, Colección Regional, Instituto Nacional de Antropología e Historia, Centro INAH-Puebla, Puebla de Zaragoza, Puebla.

\section{2. Área de estudio y estratigrafía local}

El material descrito en este trabajo proviene de dos localidades en la región centro-este del estado de Puebla. La primera localidad se denomina barranca El Morillo y se localiza a $2 \mathrm{~km}$ al norte de Ciudad Serdán, municipio de Chalchicomula de Sesma (Figura 1). En este sitio aflora una secuencia de sedimentos fluviolacustres de $4.5 \mathrm{~m}$ de espesor, donde se observan tres secciones litológicas diferentes. La inferior con un espesor de $2.9 \mathrm{~m}$, está conformada por arcilla con una fracción de arena fina que intemperiza a color café claro y en donde, además, hay presencia de pequeños lentes de ceniza volcánica de entre 15 a $20 \mathrm{~cm}$ de espesor; este estrato es donde se encontró el material de Mammuthus correspondiente al ejemplar CRINAH-2275. A su vez este estrato de arcilla es sobreyacido por un estrato de arena gruesa de color gris oscuro de aproximadamente $1 \mathrm{~m}$ de espesor, donde están presentes litoclastos subredondeados de cuarzo, sienita, basalto, andesita y granito de tamaños que van desde $5 \mathrm{~cm}$ hasta $20 \mathrm{~cm}$ de diámetro. El estrato superior tiene $60 \mathrm{~cm}$ de espesor y está conformado por arena fina con alto contenido de materia vegetal. En esta sección hay presencia de paleocanales, los cuales están rellenos por arena gruesa que contienen además pequeños litoclastos rodados de cuarzo, calcita, basalto, granito y andesita, que van de 0.50 a $2 \mathrm{~cm}$ de diámetro (Figura 2A).

La segunda localidad se denomina Sierra de Amozoc y se localiza a $3.5 \mathrm{~km}$ al sur de Amozoc de Mota, en la ladera oriente de una loma a 40 me- tros del antiguo camino a Cuautinchan, en las inmediaciones de la colonia Las Cruces (Figura 1). La secuencia estratigráfica expuesta mide $2.20 \mathrm{~m}$ de espesor y se observan tres niveles sedimentarios. El estrato inferior tiene $1.40 \mathrm{~m}$ de espesor y está conformado por arena de grano medio y toba andesítica de color ocre muy consolidada; en esta sección se encontraron los restos de Mammuthus que corresponden al ejemplar CRINAH-2274. La sección inmediata superior está conformada por un estrato de aproximadamente $45 \mathrm{~cm}$ de arena gruesa a mediana con pequeños litoclastos subredondeados de rocas volcánicas. Finalmente, la secuencia culmina con un estrato de $50-55 \mathrm{~cm}$ de arena fina y limo que presenta un alto contenido de materia vegetal (Figura 2B).

\section{Material y método}

\subsection{MATERIAL}

Se describen dos ejemplares; el primer ejemplar fue recolectado en Sierra de Amozoc y está catalogado como CRINAH-2274 y comprende un cráneo parcialmente deformado que conservó en su mayoría la parte derecha; el M2 derecho, el M2 y M3 izquierdos y parte de la defensa izquierda en mal estado de conservación. El segundo ejemplar fue rescatado en la barranca El Morillo y está catalogado como CRINAH-2275, e incluye el M2 y M3 derechos, M2 y M3 izquierdos; fémur derecho al cual le falta el extremo proximal y distal; tibia izquierda parcialmente conservada, vértebras incompletas y varios fragmentos de costillas. Ambos ejemplares están resguardados en el laboratorio de Paleontología del Centro INAH-Puebla, del Instituto Nacional de Antropología e Historia.

\subsection{OBTENGIÓN DE MEDIDAS Y FOTOGRAFÍAS}

Las medidas craneales se obtuvieron siguiendo a Gillette y Madsen (1993), mientras que las medidas dentales y postcraneales se sacaron siguiendo las recomendaciones de Agenbroad (1994). Los huesos de gran talla fueron medidos con una cinta 


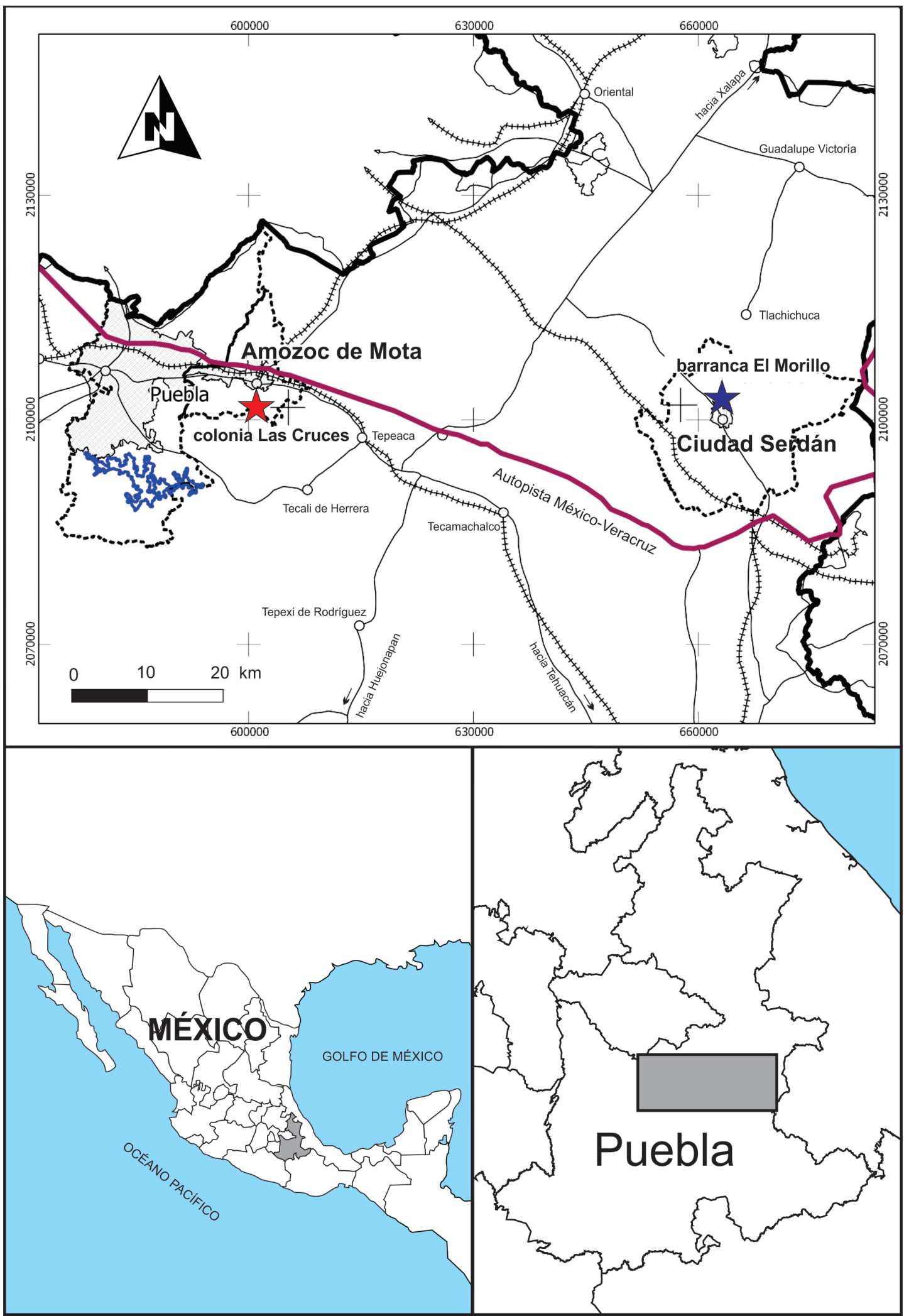

Figura 1 Ubicación de las localidades con restos de Mammuthus columbi descritos en este trabajo. La estrella azul marca la ubicación de la localidad barranca El Morillo, cerca de Ciudad Serdán; la estrella roja marca la ubicación de la localidad Sierra de Amozoc, en las inmediaciones de la colonia Las Cruces, municipio de Amozoc de Mota. 

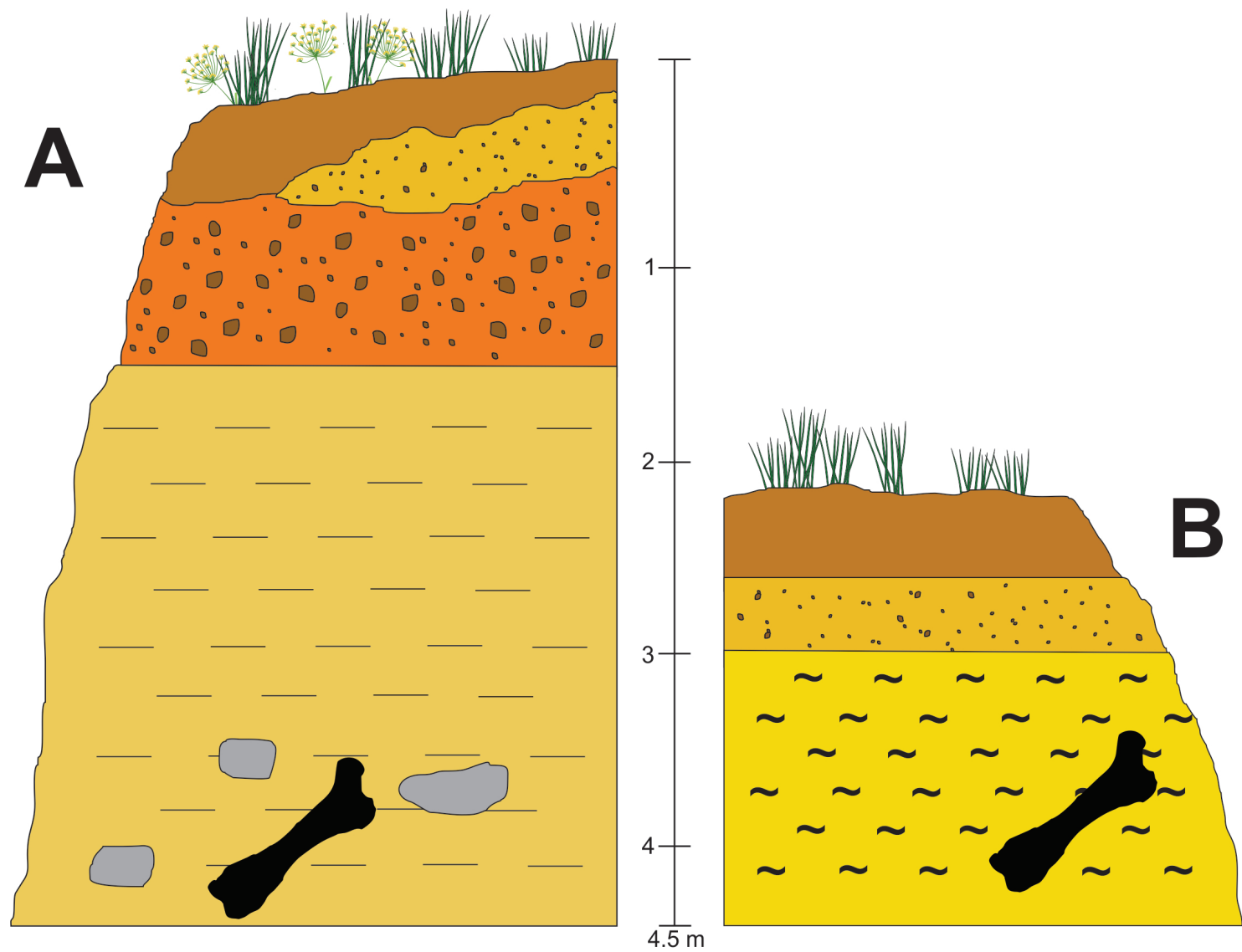

\section{Simbología}

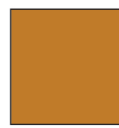

Arena de grano medio con abundante matería vegetal

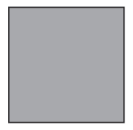

Ceniza vólcanica

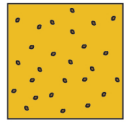

Arena de grano grueso con pequeños

litoclastos rodados
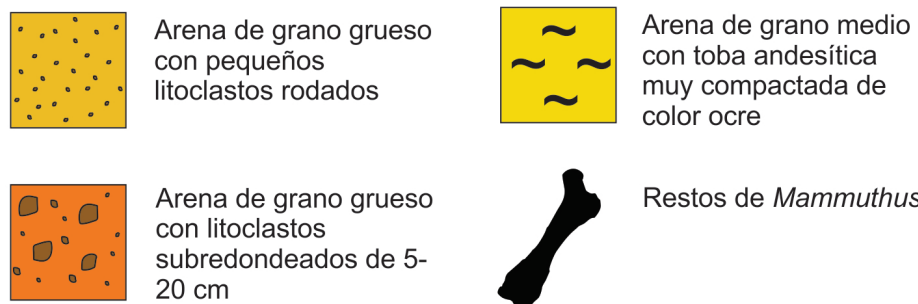

Arena de grano grueso con litoclastos

subredondeados de 5 $20 \mathrm{~cm}$
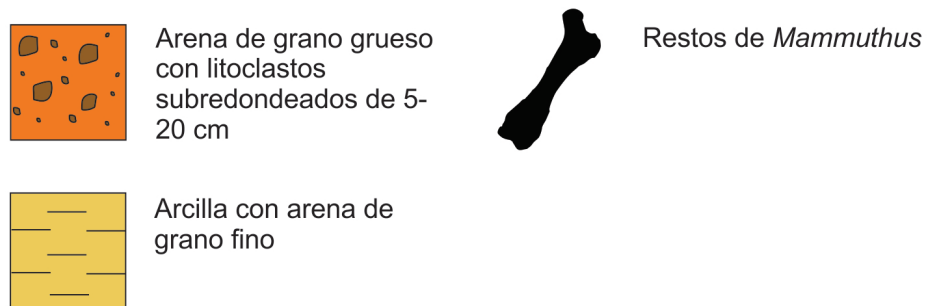

Arcilla con arena de grano fino 
métrica. Las medidas dentales se obtuvieron con un calibrador digital con una precisión de $0.1 \mathrm{~mm}$. En las Figuras 3 y 4 se muestra cómo se tomaron las medidas del cráneo y molares.

Se obtuvo la frecuencia lamelar de cada molar; esta se refiere al número promedio de placas en
$100 \mathrm{~mm}$ tomados a lo largo del eje anteroposterior en la superficie oclusal del molar (Maglio, 1973).

Las fotografías se obtuvieron con una cámara digital Canon PowerShot SX40 HS de 12.1 megapixeles y fueron procesadas en CorelDraw X8.
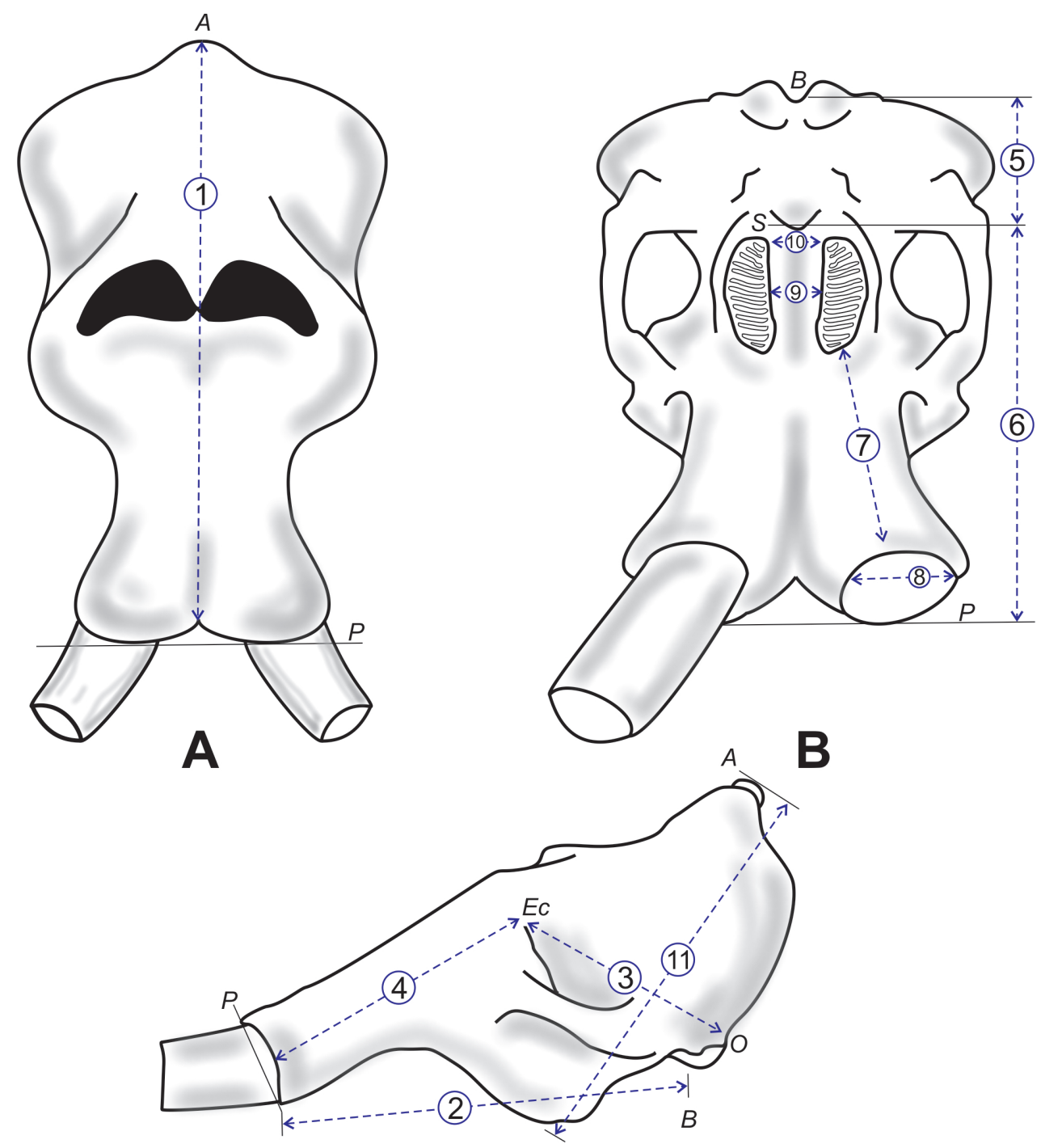

C

Figura 3 Esquema que muestra cómo se tomaron las medidas en el cráneo: 1) longitud total: proyectado sobre la línea media (acrocraniónprostión); 2) longitud basal (basión-prostión; 3) longitud de la caja craneal (ectorbital-opisthion); 4) longitud facial lateral (ectorbitalprostión); 5) longitud basión-staphilion; 6) longitud del paladar (staphilion-prostión; 7) longitud del diastema: desde el alveolo del M3 hasta el alveolo del incisivo (medido desde el punto medio del alveolo); 8) diámetro transverso interno del alveolo del incisivo; 9) ancho máximo de la curvatura del maxilar en el punto medio del alveolo del M3; 10) ancho máximo posterior del paladar: medido desde el extremo posterior de los alvéolos del M3; y 11 ) altura del cráneo: desde el paladar hasta el acrocranión. Abreviaturas: A, acrocranión; B, basión; Ec, ectorbital; O, opisthion; P, prostión; S, staphilion. Modificado de Gillette y Madsen (1993). 


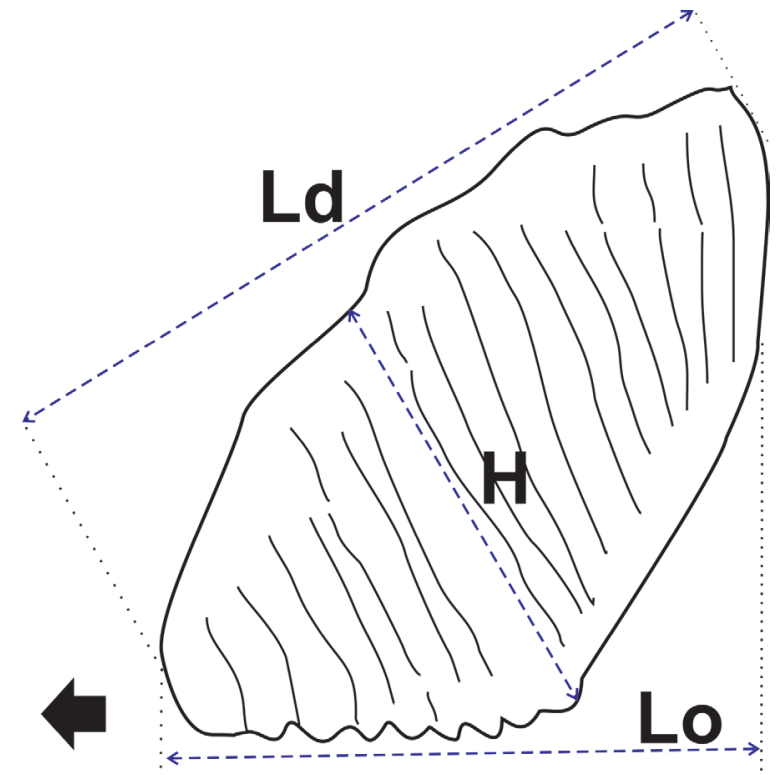

Figura 4 Esquema que muestra cómo se tomaron las medidas en los molares. Abreviaturas: $\mathrm{H}$, altura máxima del diente paralela a la orientación de las láminas; Ld, longitud máxima en plano diagonal; Lo, longitud máxima en el plano oclusal. Modificado de Agenbroad (1994).

\subsection{NOMENGLATURA GRANEAL Y DENTAL}

En elefantes los primeros tres dientes son premolares deciduos y aunque las láminas dentales de los premolares permanentes están presentes en el epitelio oral, estos no se desarrollan (Kozawa et al., 2001). Por consiguiente, aunque existen varios sistemas nomenclaturales para los molariformes de Elephantidae, nosotros preferimos el propuesto por Saunders (1970), por lo que nombramos la serie como dP2, dP3, dP4, M1, M2, y M3, ya que consideramos se apega más a la nomenclatura tradicional de mamíferos; contrariamente al propuesto por Laws (1966), que nombra a los molariformes como M1, M2, M3, M4, M5 y M6, en la cual el dP2, dP3 y dP4 son nombrados como M1, M2 y M3 respectivamente.

La nomenclatura morfológica usada en la descripción del cráneo está basada en Van der Merwe et al. (1995).

\subsection{ESTIMAGIÓN DE LA EDAD}

La edad de los ejemplares se calculó siguiendo a
Haynes (1991) y se expresa en Años de Elefante Africano (AEA), según la escala de Laws (1966).

\subsection{INFERENGIA DE LA DIETA}

Para evaluar la dieta de los ejemplares se usó la metodología de ángulos de mesodesgaste para elefántidos propuesta por Saarinen et al. (2015), la cual consiste en medir el ángulo formado por las paredes de esmalte al interior del valle de tres láminas en uso; con estas tres medidas se obtiene la media. Los ángulos por arriba de $130^{\circ}$ indican una dieta estrictamente pacedora $>90 \%$ de plantas $\mathrm{C}_{4}$ ); de $130^{\circ}$ a $124^{\circ}$ la dieta es dominada por pastos, pero hay ingesta de plantas arbustivas (> $70 \%$ de plantas $\mathrm{C}_{4}$ ); de $124^{\circ}$ a $113^{\circ}$ la dieta es mixta (ingesta casi igual de plantas $\mathrm{C}_{3}$ y $\mathrm{C}_{4}$ ); de $113^{\circ}$ a $106^{\circ}$ la dieta es dominantemente ramoneadora, pero hay ingesta de pastos $>70 \%$ de plantas $\mathrm{C}_{3}$ ); y los ángulos por debajo de los $106^{\circ}$ indican dieta estrictamente ramoneadora $>90 \%$ de plantas $\mathrm{C}_{3}$ ).

Los ángulos se obtuvieron con un medidor de contornos, el cual se posicionó sobre la superficie oclusal de cada molar para obtener el relieve de los valles de las láminas. Posteriormente, se midió el ángulo del contorno de tres láminas en uso y se obtuvo la media (Figura 5).

\section{Resultados}

\subsection{PALEONTOLOGÍA SISTEMÁTICA}

Clase Mammalia Linnaeus, 1758 Superorden Paenungulata Simpson, 1945

Orden Proboscidea Illiger, 1811

Familia Elephantidae Gray, 1821

Género Mammuthus Brookes, 1828

Mammuthus columbi (Falconer, 1857)

Holotipo. $\mathrm{BM}(\mathrm{NH}) 40769$, M3 incompleto con 10 láminas conservadas, colectado en el Canal de Brunswick, cerca de Darien Georgia, en el año de 1838 durante la excavación del canal y actualmente alojado en el British Museum of Natural His- 


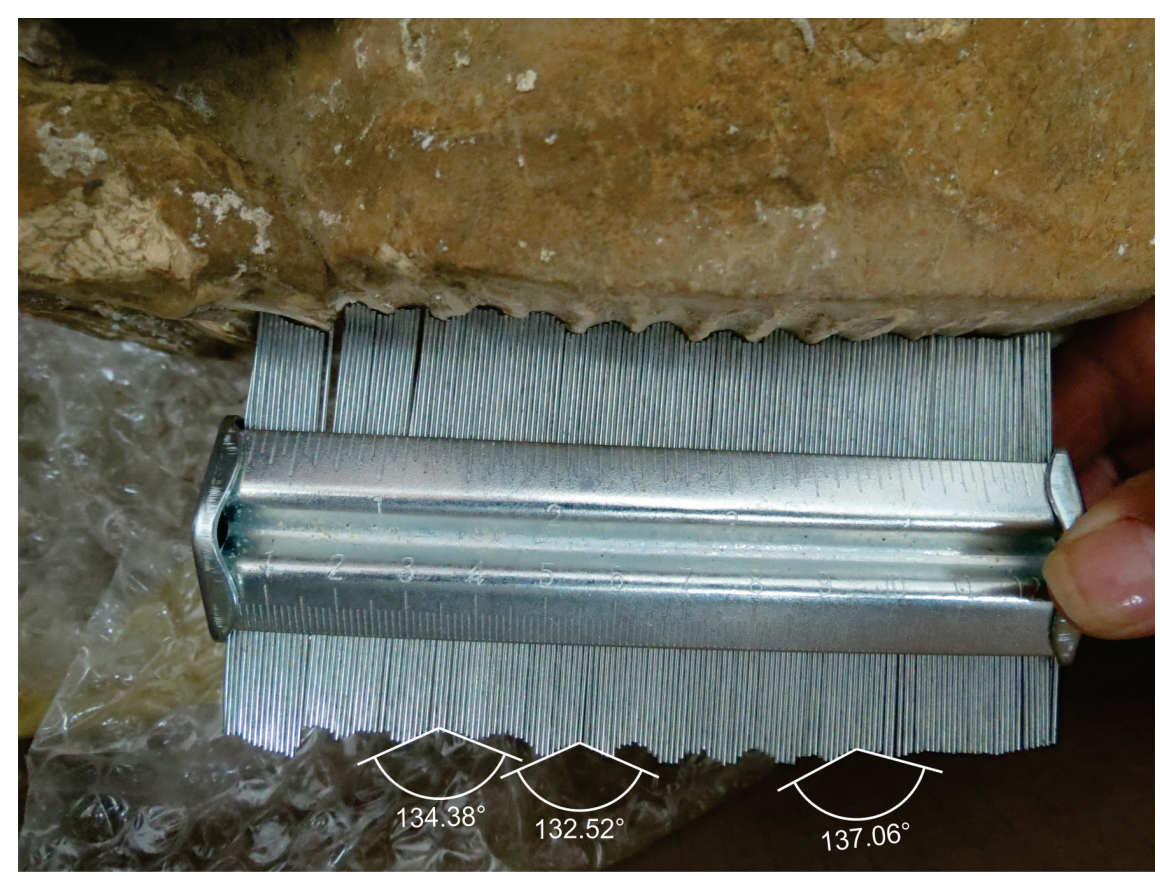

Figura 5 Uso del medidor de contornos para obtener el relieve oclusal de los molares de Mammuthus columbi, y poder calcular el ángulo interno de los valles de las láminas en uso.

tory (Maglio, 1973; Patterson et al., 2012).

Sinonimias. Archidiskodon imperator (Leidy, 1858), Elephas (Euelephas) imperator (Leidy, 1858), Euelephas imperator (Leidy, 1858), Mammuthus imperator (Leidy, 1858), Mammuthus (Archidiskodon) imperator (Leidy, 1858), Elephas jeffersonii (Osborn, 1922), Parelephas roosevelti (Hay, 1922), Elephas washingtonii (Osborn, 1923), Parelephas progressus (Osborn, 1924), Elephas eellsi (Hay, 1926), Archidiskodon sonoriensis (Osborn, 1929), Elephas floridanus (Osborn, 1929), Parelephas columbi (Osborn, 1929), Parelephas floridanus (Osborn, 1929), Parelephas jeffersonï (Osborn, 1930).

\subsection{DESGRIPGIÓN}

\subsubsection{EJEMPLAR CRINAH-2274}

El cráneo es más alto que ancho, con el frontal y el parietal extendiéndose posterodistalmente (Figura 6A). La parte posterior del parietal y supraoccipital están perdidos y permiten visualizar la neumatización interior del cráneo. Sólo está presente el meatus acusticus externus derecho y este se observa como una abertura de forma circular que se ubica por detrás del arco zigomático. El arco zigomático derecho está completo y se extiende desde la parte anterior del M2 hasta la parte posterior del M3. La facies orbitalis es profunda y se encuentra bordeada en su parte superior por el margo supraorbitalis. Únicamente está conservada la narina externa derecha, la cual se aprecia como una abertura de forma semilunar que se ubica en la parte frontal del cráneo. En la parte posterior se observa el $f_{0}$ ramen magnum, el cual es amplío y de forma circular (Figura 6B). El processus alveolaris derecho está conservado parcialmente y se orienta anterolateralmente, lo que indica que ambos incisivos son divergentes. El alveolo del incisivo derecho no está completo, pero la parte conservada permite estimar su diámetro en $210 \mathrm{~mm}$ (Tabla 1). Ventralmente se observan ambos palatinos pareados, estos son una lámina delgada orientada anteroposteriormente. Ambos palatinos se fusionan lateralmente con los procesos palatinos del maxilar. En la parte posterior de cada palatino se observa el foramen palatinum major. El vómer es una lámina delgada que divide el piso de la cavidad nasal y el techo del pasaje nasofaríngeo. Ambos canalis infraorbitalis están presentes y se localizan en la parte anterior 

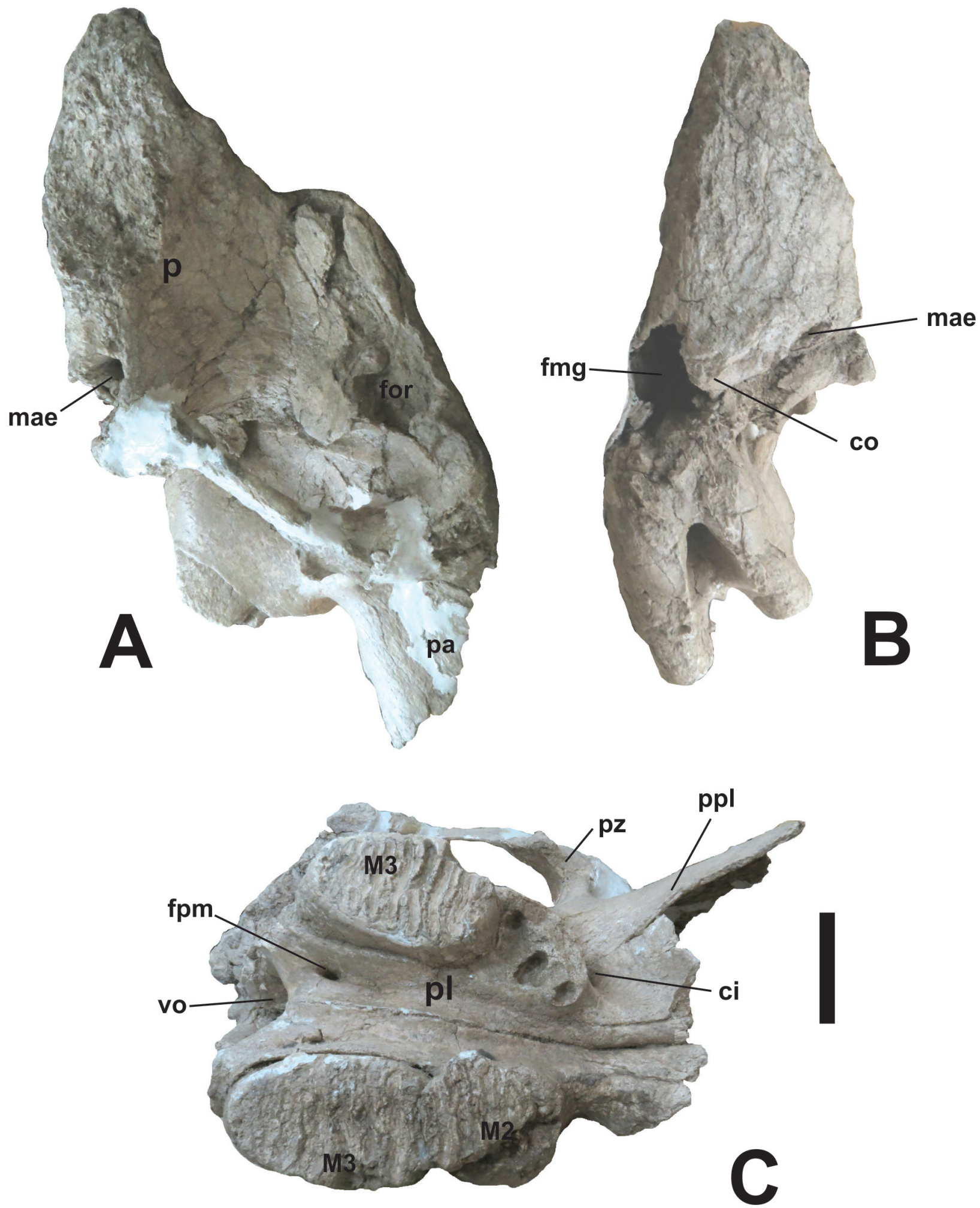

Figura 6 Ejemplar CRINAH-2274, de Sierra de Amozoc, Puebla. Cráneo en vista A) lateral (lado derecho), B) posterior y C) ventral. Barra de escala igual a $200 \mathrm{~mm}$. Abreviaturas: ci, canalis infraorbitalis; co, condylus occipitalis; fmg, foramen magnun; for, facies orbitalis; fpm, foramen palatinum major, mae, meatus acusticus externus; p, parietal; pa, processus alveolaris; pl, palatino; ppl, processus palatinus; pz, processus zygomaticus; vo, vómer. 
Tabla 1. Medidas del cráneo CRINAH-2274.

\section{Dimensiones}

1. Longitud total: proyectado sobre la línea media (acrocranión-prostión).

2. Longitud basal (basión-prostión).

3. Longitud de la caja craneal (ectorbital-opisthion).

4. Longitud facial lateral (ectorbital-prostión).

5. Longitud basión-staphilion

6. Longitud del paladar (staphilion-prostión).

7. Longitud del diastema: desde el alveolo del M3 hasta el alveolo del incisivo (medido desde el punto medio del alveolo).

8. Diámetro trasverso interno del alveolo del incisivo.

9. Ancho máximo de la curvatura del maxilar en el punto medio del alveolo del M3.

10. Ancho máximo posterior del paladar: medido desde el extremo posterior de los alvéolos del M3.

11. Altura del cráneo: desde el paladar hasta el acrocranión.

\section{$\mathbf{m m}$}

1250

850

410

700

290

680

260

220

100

120

810 de los alvéolos de los M2.

Están conservados el M2 izquierdo y el M3 derecho e izquierdo. El M2 es cuadrangular, sus medidas son $90 \mathrm{~mm}$ de largo por $90 \mathrm{~mm}$ de ancho; solamente cinco láminas están presentes. Los M3 poseen 22 láminas (Figura 6C). La superficie oclusal en uso de los M3 presenta nueve láminas y tienen entre 140 - $150 \mathrm{~mm}$ de largo y $90-100 \mathrm{~mm}$ de ancho. La frecuencia lamelar es de 6 a 7 . Tanto en el M2 como los M3 las láminas son delgadas. El esmalte es fuertemente crenulado, con un grosor de 1.5 a 4 mm.

El fragmento de defensa está muy mal conservado, pero se aprecia que corresponde a la porción proximal y tiene una longitud de $1050 \mathrm{~mm}$.

\subsubsection{EJEMPLAR GRINAH-2275}

El M2 derecho está fuertemente desgastado y sólo conserva tres láminas; su forma es cuadrangular y mide $80 \mathrm{~mm}$ de longitud por $89 \mathrm{~mm}$ de ancho. En el M3 derecho la superficie en uso mide $126 \mathrm{~mm}$ de largo por $91 \mathrm{~mm}$ de ancho; posee un total de 20 láminas, de las cuales ocho están en uso. Por su parte, la superficie oclusal del M2 izquierdo tiene una longitud de $115 \mathrm{~mm}$ y $84 \mathrm{~mm}$ de ancho; conserva únicamente tres láminas (Tabla 2). El M3 iz- quierdo posee 21 láminas de las cuales ocho están en uso. En todos los molares las láminas son delgadas; el esmalte es crenulado, con un grosor de 2.0 a $3.5 \mathrm{~mm}$ (Figura 7). Frecuencia lamelar de seis láminas en $100 \mathrm{~mm}$.

La tibia únicamente conserva la diáfisis, ya que los extremos distal y proximal están perdidos. La diáfisis es de forma suboval en corte transversal, pero hacia los extremos se ensancha abruptamente en sentido lateral-medial; el extremo proximal es más ancho que el distal.

Sólo se conservó la parte proximal de la diáfisis del fémur, la cual es de forma tubular. La forma y proporciones indican que se trata de un hueso largo, pero delgado.

Tabla 2. Medidas (en mm) de los M3 del ejemplar CRINAH 2275.

\begin{tabular}{|c|c|c|c|}
\hline Molar & Ld & Lo & H \\
\hline M3 derecho & 260 & 208 & 199 \\
\hline M3 izquierdo & 220 & 170 & 180 \\
\hline
\end{tabular}

Abreviaturas: $\mathrm{H}$, altura máxima del diente paralela a la orientación de las láminas; Ld, longitud máxima en plano diagonal; Lo, longitud máxima en el plano oclusal. 

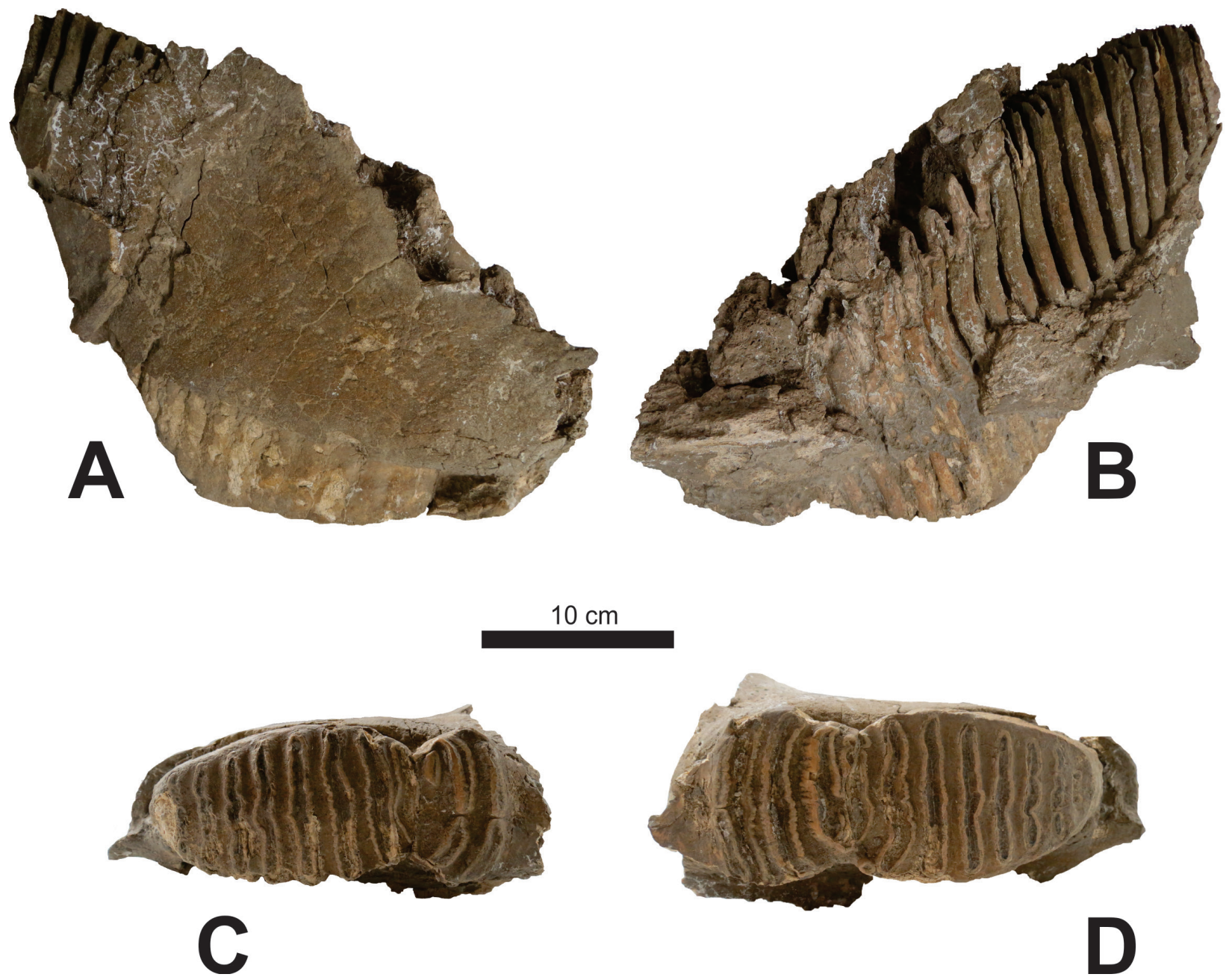

Figura 7 Ejemplar CRINAH-2275, de Barranca El Morillo, Puebla. M2 y M3 derechos en vista A) labial, B), lingual y C) oclusal; D) M2 y M3 izquierdos en vista oclusal. Barra de escala igual a $100 \mathrm{~mm}$.

\subsection{EDAD}

La edad de Mammuthus puede ser estimada usando el tamaño y grado de desgaste de los molares (Haynes, 1991), o el grado de fusión entre la epífisis y diáfisis en huesos apendiculares (Lister, 1999). Debido a que sólo un individuo posee huesos apendiculares y estos no están completos, la edad se estimó usando las variables dentales antes mencionadas.

Los M2 de M. columbi tienen un rango de 12 a 15 láminas y los M3 de 17 a 22. El ejemplar CRINAH-2274, conserva un M2 izquierdo con cinco láminas lo que indica que ha perdido entre un $60-$ $67 \%$ del total del molar. El M3 izquierdo tiene en uso ocho láminas y el M3 derecho nueve láminas de un total de 22, que muestra que los molares tienen un desgaste del 41 \%. Según Haynes (1991), estas características ubican al ejemplar en la clase XX de Laws (1966), que sugiere tiene aproximadamente 34 AEA.

El ejemplar CRINAH-2275 conserva ambos M2 y M3. En los M2 sólo están presentes tres láminas de un promedio de $12-15$, que indican la pérdida del $73 \%$ de los molares. Los M3 poseen 11 láminas en uso de las 20 totales, que muestran un desgaste en los molares del $55 \%$. Siguiendo a Haynes (1991), el ejemplar CRINAH-2275 se ubica entre las clases XXI y XXII de Laws (1966), con una edad aproximada de 37-41 AEA. 


\subsection{SEXO DE LOS INDIVIDUOS}

En Mammuthus el sexo de un individuo puede ser determinado con base en tres criterios: tamaño y robustez del esqueleto, morfología de la pelvis y dimensiones de las defensas y sus alvéolos (Lister y Agenbroad, 1994). Desafortunadamente, debido a lo incompleto de los ejemplares, sólo pudo identificarse el sexo del ejemplar CRINAH-2274, quien puede ser referido como un macho, basado en el diámetro del alveolo $(210 \mathrm{~mm})$.

\subsection{DIETA}

El valor promedio de los ángulos obtenidos en el valle interior de tres láminas en los M3 del ejemplar CRINAH-2274 fue de $136^{\circ}$ y para el M3 del ejemplar CRINAH-2275 fue de $134.5^{\circ}$. Esto indica que ambos especímenes eran pacedores estrictos con una dieta conformada principalmente por plantas $\mathrm{C}_{4}$ (Saarinen et al., 2015).

\section{Discusión}

\subsection{IDENTIDAD TAXONÓMICA}

En México llegaron a describirse varias especies de mamuts, pero actualmente sólo se reconocen dos, Mammuthus hayi que se conoce únicamente para localidades del Pleistoceno temprano (Irvingtoniano) en Arizpe, Sonora; El Mezquital, Baja California; y Culhuacán, Giudad de México (Arroyo-Cabrales et al., 2007). M. columbi se ha encontrado en sedimentos del Pleistoceno tardío (Rancholabreano), en casi todos los estados, a excepción de la Península de Yucatán y Tabasco (Arroyo-Cabrales et al., 2010). No obstante, Arroyo-Cabrales et al. (2007), mencionan que la identificación del material de M. hayi en México es cuestionable. Por su parte Lister y Sher (2015) argumentan que los M3 de $M$. hayi de El Mezquital Baja, California y Culhuacán, Ciudad de México, caen dentro del rango de medidas y frecuencia lamelar de $M$. columbi. Lo anterior cuestiona la presencia de $M$. hayi en México.
Sin embargo, nosotros preferimos reconocer conservadoramente la presencia de ambas especies a raíz de la falta de evidencia concreta que señale la inexistencia de $M$. hayi en el territorio mexicano. Los molares son una fuente altamente diagnóstica para identificar las especies dentro del género Mammuthus (Maglio, 1973), por ende, nuestra asignación se basa en la morfología y medidas de los M3 de ambos individuos. Mammuthus hayi tiene un M3 con un número bajo de láminas, 12 en total, por lo que la frecuencia lamelar es de 4; esta es la principal diferencia con el M3 de $M$. columbi que tiene una frecuencia lamelar de 5 a 7 (Arroyo-Cabrales et al., 2003a). Adicionalmente, el M3 de Mammuthus columbi tienen una longitud total de $250-320 \mathrm{~mm}$, con un ancho de 75 a $108.5 \mathrm{~mm}$; esmalte fuertemente crenulado con un grosor de 1.5 a $3.8 \mathrm{~mm}$ y poseen de 17 a 22 láminas (Arroyo-Cabrales et al., 2003a; McDaniel y Jefferson, 2006). Las características morfológicas y morfométricas de los M3 de los ejemplares CRINAH-2274 y CRINAH-2275 caen dentro del rango de los caracteres diagnósticos para $M$. columbi, por lo que ambos individuos son asignados a esta especie.

\subsection{ASPEGTOS PALEOBIOLÓGICOS}

\subsubsection{EDAD Y SEXO}

La determinación sexual y de la edad en mamíferos es importante para entender aspectos de su biología y ecología. En mamíferos extintos la determinación de estos aspectos biológicos es posible sólo en algunos casos. Por ejemplo, en Mammuthus se ha podido asignar edad y sexo a individuos equiparándolo con las especies de elefantes vivientes (Haynes, 1991).

En las especies de elefantes actuales hay diferencia de tamaño entre machos y hembras. En Loxodonta africana, los machos llegan a pesar de 4700 a 6048 kg y alcanzan una altura hasta los hombros de 3.20 a $4.01 \mathrm{~m}$, mientras que las hembras tienen rangos de 2160 a $3232 \mathrm{~kg}$ y altura hasta los hombros de 2.20 a 2.60 m (Laursen y Bekoff, 1978). En Elephas maximus, los machos llegan a pesar hasta $5400 \mathrm{~kg}$ y alcanzan tallas de $3.20 \mathrm{~m}$ hasta lo hombros, mientras que las hembras llegan a pesar en promedio 
$2720 \mathrm{~kg}$ y medir $2.24 \mathrm{~m}$ hasta los hombros (Shoshani y Eisenberg, 1982). En Mammuthus columbi estas diferencias de talla también están presentes, siendo los machos más grandes que las hembras, tal como lo han demostrado estudios que incluyen restos de varios ejemplares encontrados en un mismo yacimiento (e.g. Lister y Agenbroad, 1994; McDaniel y Jefferson, 2006). En este sentido, las dimensiones de las defensas y sus alvéolos son de gran valor para la determinación del sexo (Lister y Agenbroad, 1994), ya que los machos tienen defensas más robustas, por lo que es posible separar machos de hembras basados en el diámetro de éstas (Vereshchagin y Tikhonov, 1986).

En el caso de los ejemplares descritos en este trabajo, sólo el ejemplar CRINAH-2274 pudo ser referido como un macho con base al diámetro del alveolo derecho. La dimensión del alveolo de CRINAH-2274 es de $210 \mathrm{~mm}$. Esta medida es similar al del ejemplar apodado "Zed" colectado en Rancho La Brea, el cual tiene un diámetro de $240 \mathrm{~mm}$, pero es ligeramente mayor al del individuo con número de entrada 88.18 de la Utah Division of State History, el cual es de $190 \mathrm{~mm}$; ambos ejemplares referidos como machos (Gillette y Madsen, 1993; El Adli et al., 2015).

Los machos de Elephas y Loxodonta alcanzan la madurez sexual alrededor de los 15 años, aunque generalmente llegan a aparearse hasta los 30 años (Flower, 1943; Sukumar, 2003). La edad estimada para el ejemplar CRINAH-2274 es de 34 AEA y por lo tanto es un macho sexualmente maduro. En elefantes africanos actuales, los machos sexualmente maduros son excluidos de los grupos familiares dominados por hembras, por lo que viven solos o formando pequeños grupos con otros machos, excepto en las temporadas de apareamiento (Haynes, 1991; Moss y Poole, 1983). Esto podría explicar por qué cuando se encuentran restos de ejemplares solitarios de Mammuthus generalmente corresponden a machos adultos (e.g. Gillette y Madsen, 1993), como es el caso de CRINAH-2274.

Por su parte, el ejemplar CRINAH-2275 tiene una edad de entre 37 a 41 AEA, pero no presenta caracteres que permitan determinar su sexo. No obstante, como el ejemplar fue encontrado solo, podría también tratarse de un macho sexualmente maduro.

\subsubsection{DIETA}

Saarinen et al. (2015) encontraron que en las especies actuales de proboscídeos existe una estrecha relación entre los valores isotópicos de $\delta^{13} \mathrm{C}$ (que depende de la dieta) y el ángulo formado entre los valles de las láminas en uso de los molares. Basados en sus resultados, estos autores determinaron ángulos de mesodesgaste, método con el que es posible conocer la dieta de elefántidos extintos (ver apartado Material y Método). Los ejemplares CRINAH-2274 y CRINAH-2275 tienen un ángulo promedio de $135^{\circ}$, que indican que la dieta de ambos individuos estuvo basada principalmente en pastos $\left(>90 \%\right.$ de plantas $\left.\mathrm{C}_{4}\right)$. Esta dieta es similar al encontrado en los individuos de Rancho La Brea, California, los cuales tienen un promedio de $135.5^{\circ}$ y se consideran estrictamente pacedores (Saarinen et al., 2015). Una dieta pacedora, basada en juncia (Carex spp.) y pastos, al parecer es característica en individuos de $M$. columbi procedentes de localidades rancholabreanas en Estados Unidos de América, tal como lo demuestran análisis isotópicos de los ejemplares de Ichetucknee, Florida; Ingleside, Texas; y Gila Pueblo, Arizona (Cerling et al., 1999); y análisis de heces procedentes de Salt Lake Meridian y Bechan Cave, Utah (Mead et al., 1986; Gillette y Madsen, 1993).

En México, la dieta de individuos M. columbi, provenientes de diversas localidades del centro y sur del país, ha sido determinada usando isótopos estables de $\delta^{13} \mathrm{C}$ y $\delta^{18} \mathrm{O}$ extraídos del esmalte dental, así como con unos pocos análisis de microdesgaste. En este trabajo, la determinación de la dieta de mamuts usando el método de ángulos de mesodesgaste se aplica por primera vez en México.

Los resultados obtenidos por los métodos arriba mencionados demuestran que la mayoría de los ejemplares tenían una dieta mixta basada en plantas $\mathrm{C}_{3} \mathrm{y}_{4}$, mientras que un menor número de individuos eran estrictamente pacedores con una ingesta exclusiva de plantas $\mathrm{C}_{4}$. Lo anterior 
se ha documentado en las localidades de El Cedral y Laguna de Las Cruces, en San Luis Potosí (Pérez-Crespo et al., 2009, 2013); Tocuila, Estado de México (Pérez-Crespo et al., 2010); Valsequillo, Puebla (Pérez-Crespo et al., 2014); La Cinta-Portalitos y La Piedad-Santa Ana, en el límite Michoacán-Guanajuato (Gutiérrez-Bedolla et al., 2016); El Amate, en la Depresión Central, Chiapas (Pérez-Crespo et al., 2015); y ahora en barranca El Morillo y Sierra de Amozoc, Puebla.

\subsubsection{PREFERENGIA DE HÁBITAT}

La inferencia de la dieta de mamíferos herbívoros extintos ha sido frecuentemente usada como un indicador de la vegetación presente en un área y tiempo determinados, ya que la ingesta de alimento está ligada estrechamente a las plantas que se encuentran disponibles en el entorno. Esto a su vez da una aproximación de las condiciones paleoambientales existentes del área geográfica en un determinado intervalo de tiempo (Rivals y Athanassiou, 2008), debido a que las plantas poseen vías metabólicas variadas y, por consiguiente, tienen requerimientos abióticos específicos para su desarrollo (Koch, 1998). La inferencia de la dieta de individuos de $M$. columbi de las localidades estadounidenses y mexicanas ha permitido tener una idea de las condiciones paleoambientales en los que estos vivían. La información disponible indica que $M$. columbi en Estados Unidos de América y México habitaba zonas abiertas con abundancia de plantas herbáceas, en los que había presencia de árboles bajos, donde además predominaban climas secos y fríos. En consecuencia, con base en la determinación de la dieta de los ejemplares CRINAH-2274 y CRINAH-2275, es posible inferir que durante el Pleistoceno en las localidades Barranca el Morillo y Sierra de Amozoc, existieron zonas abiertas con pastos. Este paleoambiente podría ser equiparado al existente durante el Pleistoceno en la Cuenca de Valsequillo, Puebla, zona que se ubica a tan sólo $78 \mathrm{~km}$ de barranca El Morillo y a $22 \mathrm{~km}$ de Sierra de Amozoc. La reconstrucción del paleoambiente de la Cuenca de Valsequillo indica predominio de vegetación abierta, pero con presencia de coníferas (Pinus, Quercus, Alnus), donde predominaba un clima de baja humedad, que alternaba episodios secos y fríos (Stevens et al., 2012; Pérez-Crespo et al., 2014; Metcalfe et al., 2016). Por consiguiente, es posible que este tipo de hábitat fuera común en el centro del estado de Puebla durante el Pleistoceno, favoreciendo el desarrollo de las poblaciones de $M$. columbi.

\section{Conclusiones}

La descripción del material de proboscídeos catalogados como CRINAH-2274 y CRINAH-2275, procedente de las localidades barranca El Morillo y Sierra de Amozoc, permitió la identificación del mamut Mammuthus columbi, adicionando así nuevos registros de esta especie en México. Asimismo, pudo establecerse que ambos ejemplares eran adultos sexualmente maduros. Únicamente se determinó el sexo para el individuo CRINAH-2274, basado en el diámetro del alveolo del incisivo, cuyas dimensiones son similares a las de ejemplares de Estados Unidos de América, que permitió referirlo como un macho.

La determinación de la edad y sexo en $M$. colum$b i$ se determinó por primera vez para ejemplares procedentes del estado de Puebla. Con esto se contribuye al conocimiento de algunos aspectos paleobiológicos de esta especie en México, que sin duda servirán para entender mejor la dinámica poblacional de $M$. columbi en América del Norte. Por último, se aplicó por primera vez en México el análisis de ángulos de mesodesgaste. Con este método se determinó que la dieta de los ejemplares CRINAH-2274 y CRINAH-2275, fue estrictamente pacedora. Lo anterior, a su vez, permite inferir que el hábitat de ambos individuos fue un área abierta con abundancia de pastos. Esto permite tener un esbozo general sobre las condiciones paleoambientales ocurridas en la región centro-este del estado de Puebla durante el Pleistoceno. 


\section{Agradecimientos}

Queremos expresar nuestro agradecimiento a Francisco Ortiz Pedraza, delegado del centro INAH-Puebla, y a Delia Domínguez Cuanalo, por las facilidades que nos otorgaron para la realización de este trabajo en el Laboratorio de Paleontología del mismo Centro. A la familia Valdés Bonilla por su interés y apoyo durante el tiempo que duraron las excavaciones del mamut de la Sierra de Amozoc, así como a las autoridades municipales de Amozoc de Mota, Puebla, por el apoyo incondicional que nos ofrecieron durante los trabajos de rescate. A Felicitas Rojas y Jair Peña Serrano quienes participaron en el rescate del ejemplar de la barranca El Morillo, Ciudad Serdán, municipio de Chalchicomula de Sesma. También agradecemos de manera especial a Raymundo Flaviano Rodríguez Chávez y Judith Romero Rosas por su apoyo durante la consulta de los materiales en el depósito de Bienes del centro INAH-Puebla, y a Luis Enrique Gómez-Pérez por los comentarios que ayudaron a mejorar la redacción del manuscrito. Por último, agradecemos a los revisores Víctor Adrián Pérez-Crespo, César A. Laurito y Víctor M. Bravo-Cuevas, así como al comité editorial del Boletín, por las sugerencias vertidas, las cuales enriquecieron sustancialmente el trabajo.

\section{Referencias}

Agenbroad, L.D., 1994, Taxonomy of North American Mammuthus and biometrics of the Hot Springs Mammoths, en Agenbroad, L.D., Mead, J.I. (eds.), The Hot Springs Mammoth Site: A decade of field and laboratory research in paleontology, geology, and paleoecology: South Dakota, The Mammoth Site of Hot Springs, 158-207.

Alvarado, G., 1986, Hallazgo de megamamíferos fósiles en Costa Rica: Revista Geológica de América Central, 4, 1-46.
Armenta-Camacho, J., 1957, Hallazgos prehistóricos en el valle de Puebla, 2: Centro de Estudios Históricos de Puebla.

Armenta-Camacho, J., 1978, Vestigios de labor humana en huesos de animales extintos de Valsequillo, Puebla, México: Publicaciones del Consejo Editorial del Gobierno del Estado de Puebla, 126 p.

Arroyo-Cabrales, J., Polaco, O.J., Aguilar-Arellano, F., 2003a, Remains of the Mammuthus housed in the collections of the Instituto Nacional de Antropología e Historia, México, en Reumer, J.W.F., De Vos, J., Mol, D. (eds.), Advances in Mammoth research, Proceedings of the Second International Mammoth Conference, Rotterdam, May 16-20, 1999: Deinsea, 9, 17-25.

Arroyo-Cabrales, J., Polaco, O.J., Johnson, E., Guzmán, A.F., 2003b, The distribution of the genus Mammuthus in Mexico, en Reumer, J.W.F., De Vos, J., Mol, D. (eds.), Advances in mammoth research, Proceedings of the Second International Mammoth, Conference, Rotterdam, May 16-20, 1999: Deinsea, 9, 27-39.

Arroyo-Cabrales, J., Polaco, O.J., Laurito, C., Johnson, E., Alberdi, M.T., Valerio Zamora, A.L., 2007, The proboscideans (Mammalia) from Mesoamerica: Quaternary International, 169-170, 17-23.

Arroyo-Cabrales, J., Polaco, O.J., Johnson, E., Ferrusquía-Villafranca, I., 2010, A perspective on mammal biodiversity and zoogeography in the Late Pleistocene of México: Quaternary International, 212(2), 187-197.

Brookes, J., 1828, A catalogue of the anatomical zoological museum of Joshua Brookes, Esq., F.R.S., F.L.S. \&c. Part 1: London, Richard Taylor, $76 \mathrm{p}$.

Cerling, T.E., Harris, J.M., Leakey, M.G., 1999, Browsing and grazing in elephants: the isotope record of modern and fossil proboscideans: Oecologia, 120, 364-374. 
El Adli, J.J, Cherney,M.D., Fisher, D.C., Harris, J.M., Farrell, A.B., Cox, S.M., 2015, Last years of life and season of death of a Columbian mammoth from Rancho La Brea: Science Series, 42, 65-80.

Falconer, H., 1857, On the species of mastodon and elephant occurring in the fossil state of Great Britain: Quarterly Journal of the Geological Society of London, 13, 307-360.

Félix, J., Lenk, H., 1891, Uebersieht über die geologischen Verhältnisse des mexieanisehen Staates Puebla: Paleontogaphica in beitragezur geologic und palleontologic der republik Mexico, 37, 117-139.

Flower, S.S., 1943, Notes on age at sexual maturity, gestation period and growth of the Indian elephant, Elephas maximus: Proceeding of the Zoological Society of London, serie A, 113, 21-26.

Freudenberg, W., 1922, Die Saugetier-faunades Pliocans und Postpliocans von Mexiko-II. Mastodonten und Elefanten: Geologische und Palaeontologische Abhandlun-gen, 18, $1-76$.

Gillette, D.D., Madsen, D.B., 1993, The Columbian mammoth, Mammuthus columbi, from the Wasatch Mountains of central Utah: Journal of Paleontology, 67(4), 669-680.

Gómez-Pérez, L.E., Carbot-Chanona, G., 2012, Contribución al estudio de los megamamíferos del Pleistoceno Tardío del municipio de Villaflores, Chiapas, México: Lacandonia, 6(1), 31-41.

Gray, J.E., 1821, On the natural arrangement of vertebrose animals: London Medical Repository, 15, 296-310.

Gutiérrez-Bedolla, M., García-Zepeda, M.L., López-García, R., Arroyo-Cabrales, J., Marín-Leyva, A.H., Meléndez-Herrera, E., Fuentes-Farías, A.L., 2016, Diet and habitat of Mammuthus columbi (Falconer, 1857) from two Late Pleistocene localities in central western Mexico: Quaternary International, 406, 137-146.
Hay, O.P., 1922, Further observations on some extinct elephants: Proceedings of the Biological Society of Washington, 35, 97-102.

Hay, O.P., 1926, Description of remains of an elephant found at Port Williams, Washington: Journal of the Washington Academy of Sciences, 16(6), 154-159.

Haynes, G., 1991, Mammoths, mastodonts and elephants: Biology, behavior, and the fossil record: New York, Cambridge University Press, 413 p.

Illiger, C., 1811, Prodromus systematis mammalium et avium additis terminis zoographicis utriudque classis. C: Salfeld, Berlin, $301 \mathrm{p}$.

Irwin-Williams, C., 1967, Associations of early man with horse, camel, and mastodon at Hueyatlaco, Valsequillo (Puebla, Mexico), en Martin, P.S., Wright, H.E. Jr. (eds.), Pleistocene extinctions: the search for a cause: New Haven and London, Yale University Press, 337-347.

Koch, P.L., 1998, Isotopic reconstruction of past continental environments: Annual Review of Earth and Planetary Sciences, 26, 573-613.

Kozawa, L.H., Mishima, H., Suzuki, K., Ferguson, M.W.J., 2001, Dental formula of elephant by the development of tooth germ, en Cavarretta, G., Gioia, P., Mussi, M., Palombo, M.R. (eds.), The World of Elephants: Rome, Proceedings of the First International Congress, 639-642.

Kurtén, B., Anderson, E., 1980, Pleistocene mammals of North America: New York, Columbia University Press, 442 p.

Lagunas-Rodríguez, Z., Suárez-Cruz, S., 1997, Los restos de mamut encontrados en Tres Cerritos, Puebla: Revista Mexicana de Estudios Antropológicos, 43, 91-108.

Lagunas-Rodríguez, Z., Suárez-Cruz, S., Ocaña del Rio, B., 2013, Fósiles de proboscidios en el estado de Puebla: Dualidad, 13, 24-27. 
Lambert, D., Shoshani, J., 1998, Proboscidea, en Janis, C.M., Scott, K.M., Jacobs, L.L. (Eds.), Evolution of Tertiary mammals of North America. Vol. 1: Terrestrial carnivores, ungulates, and ungulatelike mammals: United Kingdom, Cambridge University Press, 606-621.

Laurito, G., 1988, Los proboscidios fósiles de Costa Rica y su contexto en la América Central: Vínculos, 14(1-2), 29-58.

Laurito, C., Aguilar, D., 2006, El registro de Mammuthus (Proboscidea, Elephantidae) en la República de El Salvador, América Central: Revista Geológica de América Central, 3435, 73-81.

Laursen, L., Bekoff, R., 1978, Loxodonta africana: Mammalian Species, 92, 1-8.

Laws, R.M., 1966, Age criteria for the African elephant: East African Wildlife Journal, $4,1-37$.

Leidy, J., 1858, Notice of remains of extinct vertebrata, from the Valley of the Niobrara River, collected during the exploring expedition of 1857, in Nebraska, under the command of Lieut. G. K. Warren, U. S. Top. Eng., by Dr. F. V. Hayden, geologist to the expedition: Proceedings of the Academy of Natural Sciences of Philadelphia, 10, 15-89.

Linnaeus, C., 1758, Systema Nature per regna tria naturae, secundum classes, ordines, genera, species cum characteribus, differentiis, synonymis, locis. Tomo 1, Décima edición: Estocolmo, Laurentii Salvii, 824 p.

Lister, A.M., 1999, Epiphyseal fusión and postcranial age determination in the woolly mammoth Mammuthus primigenius, en Haynes, G., Klimowicz, J., Reumer, J.W.F. (eds.), Mammoths and the mammoth fauna: Studies of an extinct ecosystem: Deinsea, 6, 79-88.

Lister, A.M., Agenbroad, L.D., 1994, Gender determination of the Hot Springs mammoths, en Agenbroad, L.D, Mead, J.I. (eds.), The Hot Springs Mammoth Site: A decade of field and laboratory research in paleontology, geology and paleoecology:
South Dakota, The Mammoth Site of South Dakota, 208-214.

Lister, A.M., Sher, A.V., 2015, Evolution and dispersal of mammoths across the Northern Hemisphere: Science, 350, 805-809.

Lucas, S., Alvarado, G., Vega, E., 1997, The Pleistocene mammals of Costa Rica: Journal of Vertebrate Paleontology, 17(2), 413-427.

Maglio, V.J., 1973, Origen and evolution of the Elephantidae: Transactions of the American Philosophical Society, 63(3), 1-149.

McDaniel Jr., G.E., Jefferson, G.T., 2006, Dental variation in the molars of Mammuthus columbi var. M. imperator (Proboscidea, Elephantidae) from a Mathis gravel quarry, southern Texas: Quaternary International, 142-143, 166-177.

Mead, J.I., Agenbroad, L.D., Davis, O.K., Martin, P.S., 1986, Dung of Mammuthus in the arid southwest, North America: Quaternary Research, 25, 121-127.

Metcalfe, S.E., Leng, M.J., Kirby,J.R., Huddart, D., Vane, C.H., Gonzalez, S., 2016, Early-Mid Pleistocene environments in the Valsequillo Basin, Central Mexico: a reassessment: Journal of Quaternary Science, 31(4), 325-336.

Moss, C.J., Poole, J.H., 1983, Relationships and social structure of African elephants, en Hinde, R.A. (ed.), Primate social relationship: Sinauer, Sunderland, Massachusetts, 315-325.

Osborn, H.F., 1905, Recent vertebrate paleontology fossil mammals of Mexico: Science, 21 (546), 931-932.

Osborn, H.F., 1922, Species of American Pleistocene mammoths Elephas jeffersonii, new species: American Museum Novitates, 41, $1-16$.

Osborn, H.F., 1923, New subfamily, generic, and specific stages in the evolution of the Proboscidea: American Museum Novitates, 99, 1-4.

Osborn, H.F., 1924, Parelephas in relation to phyla 
and genera of the family Elephantidae: American Museum Novitates, 152, 1-7.

Osborn, H.F., 1929, New eurasiatic and american proboscideans: American Museum Novitates, 393, 1-23.

Osborn, H.F., 1930, Parelephas floridanus from the Upper Pleistocene of Florida compared with P. jeffersonii: American Museum Novitates, 443, 1-17.

Osborn, H.F., 1942, Proboscidea: a monograph of the discovery, evolution, migration, and extinction of the mastodons and elephants of the world. Vol. II: Stegodontoidea, Elephantoidea: New York, The American Museum Press, 805-1675.

Patterson, D.B., Mead, A.J., Bahn, R.A., 2012, New skeletal remains of Mammuthus columbi from Glynn County, Georgia with notes on their historical and paleoecological significance: The Southeastern Naturalist, 11(2), 163-172.

Pérez-Crespo, V.A., Sánchez-Chillón, B., ArroyoCabrales, J., Alberdi, M.A., Polaco, O.J., Santos-Moreno, A., Benammi, M., MoralesPuente, P., Cienfuegos-Alvarado, E., 2009, La dieta y el hábitat del mamut y los caballos del Pleistoceno tardío de El Cedral con base en isótopos estables $\left(\delta^{13} \mathrm{C}, \delta^{18} \mathrm{O}\right)$ : Revista Mexicana de Ciencias Geológicas, 26(2), 347-355.

Pérez-Crespo, V.A., Arroyo-Cabrales,J., Benammi, M., Santos-Moreno, A., Morett-A., L., Morales-Puente, P., Cienfuegos-Alvarado, E., 2010, Carbon isotopic values of tooth enamel of Mammuthus columbi from Tocuila, State of México, México: Current Research in the Pleistocene, 27, 188-190.

Pérez-Crespo, V.A., Arroyo-Cabrales, J., Benammi, M., Polaco, O.J., Santos-Moreno, A., Morales-Puente, P., Cienfuegos-Alvarado, E., Otero, FJ., 2013, Variación de la dieta del mamut de las praderas de Laguna de las Cruces, San Luis Potosí, México: Boletín de la Sociedad Geológica Mexicana, 65(3), 573-580.
Pérez-Crespo, V.A., Arroyo-Cabrales, J., Alva-Valdivia, L.M., Morales-Puente, P., Cienfuegos-Alvarado, E., Otero, F.J., Ochoa-Castillo, P., 2014, La paleodieta de cinco especies de mamíferos herbívoros rancholabreanos de Valsequillo (Puebla, México): Revista Chilena de Antropología 30, 76-82.

Pérez-Crespo, V.A., Carbot-Chanona, G., Morales-Puente, P., Cienfuegos-Alvarado, E., Otero, FJ., 2015, Paleoambiente de la Depresión Central de Chiapas, con base en isótopos estables de carbono y oxígeno: Revista Mexicana de Ciencias Geológicas, 32(2), 273-282.

Rivals, F., Athanassiou, A., 2008, Dietary adaptations in an ungulate community from the late Pliocene of Greece: Palaeogeography, Palaeoclimatology, Palaeoecology, 265, 134-139.

Rochín-Bañaga, H., Schwennicke, T., Herrera-Gil, L.A., 2017, Registro de Mammuthus columbi en el área de El Carrizal, Baja California Sur, México: Boletín de la Sociedad Geológica Mexicana, 69(2), 495-503.

Saarinen, J., Karme, A., Cerling, T., Uno, K., Säilä, L., Kasiki, S., Ngene, S., Obari, T., Mbua, E., Manthi, F.K., Fortelius, M., 2015, A new tooth wear-based dietary analysis method for Proboscidea (Mammalia): Journal of Vertebrate Paleontology, 35(3), e918546.

Saunders, J.J., 1970, The distribution and taxonomy of Mammuthus in Arizona: Tucson, The University of Arizona, Tesis de Maestría, $115 \mathrm{p}$.

Shoshani, J., 2005, Proboscidea, en Wilson, D.E., Reeder, D.M. (eds.), Mammal species of the World. A Taxonomic and geographic references, Vol. 1, 3ra edición: Maryland, Johns Hopkins University Press, 90-91.

Shoshani, J., Eisenberg, J.F., 1982, Elephas maximus: Mammalian Species, 182, 1-8.

Shoshani, J., Tassy, P., 2005, Advances in proboscidean taxonomy and classification, anatomy and physiology, and ecology and 
behavior: Quaternary International, 126128, 5-20.

Silva-Bárcenas, S.A., 1969, Localidades de vertebrados fósiles en la República Mexicana: Paleontología Mexicana, 28, 1-34.

Simpson, G.G., 1945, The principles of classification and a classification of mammals: Bulletin of the American Museum of Natural History, 85, 1-350.

Stevens, R.E., Metcalfe, S.E., Leng, M.J., Lamb, A.L., Sloane, H.J., Naranjo, E., Silvia González, S., 2012, Reconstruction of late Pleistocene climate in the Valsequillo Basin (Central Mexico) through isotopic analysis of terrestrial and freshwater snails: Palaeogeography, Palaeoclimatology, Palaeoecology, 319-320, 16-27.

Suárez-Cruz, S., Lagunas Rodríguez, Z., 2008, Los restos de mamut de Santa Catarina Villa Nueva, Puebla, en Serrano-Sánchez, C., Cardoso-Gómez, M.A. (eds.), El Mediterráneo Americano: Población, cultura e historia, 1: México, XXVII Mesa Redonda,
Sociedad Mexicana de Antropología / Instituto de Investigaciones Antropológicas, Universidad Nacional Autónoma de México, 469-476.

Sukumar, R., 2003, The living elephants: Evolutionary ecology, behavior, and conservation: New York, Oxford University Press, 478 p.

Van der Merwe, N.J., Bezuidenhout, A.J., Seegers, C.D., 1995, The skull and mandible of the African elephant (Loxodonta africana): Onderstepoort Journal of Veterinary Research, 62, 245-260.

Vereshchagin, N.K., Tikhonov, A.N., 1986, Issledovaniya bivnei mamontov (Study of the mammoth tusks), en Vereshchagin, N.K., Kuzmina, J.Ye. (eds.), Mlekopitayushchie Chetvertichnoi Fauny SSSR (Mammals of the Quaternary Fauna of the USSR), vol. 149: Trudy Zoologicheskogo Instituta, 3-14 (en ruso). 